\title{
UCRL-JRNL-229005
}

LAW RENCE LIVERMORE N A T IO N A L LABORATORY
Improved Convergence for Two-Component Activity Expansions

H. E. DeWitt, F. J. Rogers, V. Sonnad

March 13, 2007

Physical Review E 
This document was prepared as an account of work sponsored by an agency of the United States Government. Neither the United States Government nor the University of California nor any of their employees, makes any warranty, express or implied, or assumes any legal liability or responsibility for the accuracy, completeness, or usefulness of any information, apparatus, product, or process disclosed, or represents that its use would not infringe privately owned rights. Reference herein to any specific commercial product, process, or service by trade name, trademark, manufacturer, or otherwise, does not necessarily constitute or imply its endorsement, recommendation, or favoring by the United States Government or the University of California. The views and opinions of authors expressed herein do not necessarily state or reflect those of the United States Government or the University of California, and shall not be used for advertising or product endorsement purposes. 


\title{
UCRI-JRNL-229005
}

\section{Improved Convergence for Two-Component Activity}

\section{Expansions}

\author{
H.E. DeWitt, F. J. Rogers, and V. Sonnad \\ Science and Technology Directorate, Lawrence Livermore National Laboratory, P.O. Box 808, Livermore, \\ CA, 94550
}

It is well known that an activity expansion of the grand canonical partition function works well for attractive interactions, but works poorly for repulsive interactions, such as occur between atoms and molecules. The virial expansion of the canonical partition function shows just the opposite behavior. This poses a problem for applications that involve both types of interactions, such as occur in the outer layers of low-mass stars. We show that it is possible to obtain expansions for repulsive systems that convert the poorly performing Mayer activity expansion into a series of rational polynomials that converge uniformly to the virial expansion. In the current work we limit our discussion to the second virial approximation. In contrast to the Mayer activity expansion the activity expansion presented herein converges for both attractive and repulsive systems.

PACS numbers(s): 05.20.-y $\quad$ 05.70.Ce $52.25 . \mathrm{Kn}$

\section{INTRODUCTION}


In an earlier paper [1] (hereafter referred to as I) we showed that the activity (fugacity) expansion obtained from the grand canonical ensemble is the natural procedure for obtaining the equation of state of a reacting gas as the temperature changes. In I we developed the activity expansion for the Coulomb interaction among ions and electrons. For the attractive interaction between ions and electrons in partially-ionized plasmas the activity expansion was particularly useful, but for repulsive interactions among neutral atoms it was less useful. In I we were concerned with low-density, weakly coupled hydrogen. In subsequent papers we extended the method to strongly coupled, high $\mathrm{Z}$ plasmas [2-5]. The validity of the method has been established by comparison with experiments [6-7]. It has also been shown to give good agreement with helioseismic measurements of the solar equation of state (EOS) compared to other methods [8,9]. Molecules are a minor factor in the solar EOS, but must be considered in the outer layers of lower mass stars. That is the primary motivation of the present work.

A number of methods to determine the range of convergence of activity and virial (density) expansions have been presented $[10,13]$. They show that the Mayer activity expansion has a very limited range of convergence for repulsive potentials, but fares much better for attractive potentials. The opposite is true for the virial expansion, which gives good agreement with Molecular Dynamics [14] and Monte-Carlo [15] simulations for hardspheres, but converges poorly for attractive interactions. At electron-volt temperatures, material is composed of a plasma component of positive and negative ions, which due to the electron-ion interaction, is net attractive and a repulsive component of neutral atoms and molecules. Consequently, an expansion that combines the best features 
of both expansions is needed. In the current work we show how to construct an activity expansion for the neutral component that effectively recovers the much better performing virial expansion.

There has been some earlier work dealing with how to treat the EOS of gases that involve both attractive and repulsive interactions. Friedman and Ebeling [16] proposed a method that attempts to combine the best features of both types of expansion. They divided the pressure into a density expansion part that represents the reference system and an activity expansion part that treats the deviations from the reference system. Wertheim [17] developed a more limited, two-density formalism that can be applied to reacting molecules. Neither of these methods is suitable for our purposes. Alternatively, we have developed an activity expansion method that treats the Coulomb interactions of the plasma by the usual graphical re-summation procedure. However, the terms in the activity expansion attributable to short-range interactions are regrouped into a series of rational polynomials that uniformly converge to the virial expansion [18] (hereafter referred to as II). The combined activity expansion is then solved numerically to obtain the equation of state for arbitrary states of ionization. We dealt with the plasma component in [1-5]. In the present work we are mainly concerned with developing methods that improve the convergence properties of the repulsive component. We limit our discussion to the order of the second virial approximation. This is sufficient to calculate the EOS of stars having mass greater than about 0.15 solar.

In II we showed that if terms in the activity expansion for a one-component repulsive system are grouped and summed in a specific way, it is possible to obtain an 
activity expansion that has a greatly increased range of convergence compared to the Mayer activity expansion. In Sec. II we summarize results for the one-component problem and in Sec. III we extend the method to two-component systems. In Sec. IV we study the convergence properties of the reorganized activity expansions for one and twocomponents systems.

\section{ONE VARIABLE}

The pressure of a system of $N$ particles at volume $V$ and temperature $T$ from the canonical ensemble is:

$$
\beta P(n)=n+S(n)-n(\partial S / \partial n) .
$$

In Eq. (1) $n$ is the density and $S(n)$ is the sum of the irreducible diagrams given by

$$
S(n)=\sum_{j=2}^{\infty} \frac{n^{j}}{j-1} B_{j},
$$

where the $B_{j}$ are virial coefficients.

The grand canonical ensemble gives the pressure as a sum of reducible and irreducible diagrams and is a function of the activity, $z=(2 s+1) \lambda^{-3} e^{\mu / k T}, \lambda=\left(2 \pi \hbar^{2} / m k T\right)^{1 / 2}$ is the deBroglie wavelength, $\mu$ the chemical potential, and $\mathrm{s}$ is the spin. 
In I we showed that the grand canonical pressure and density expansions in $z$ have the form:

$$
\begin{aligned}
& \beta P(z)=z+S(z)+\sum_{m=2} \frac{z}{m !}\left(\frac{\partial}{\partial z} z\right)^{m-2}\left(\frac{\partial S}{\partial z}\right)^{m} . \\
& n(z)=z \frac{\partial}{\partial z} \beta P=z+\sum_{m=1} \frac{z}{m !}\left(\frac{\partial}{\partial z} z\right)^{m-1}\left(\frac{\partial S}{\partial z}\right)^{m},
\end{aligned}
$$

with

$$
z=n \exp (-\partial S / \partial n)
$$

$S(z)$ is the sum of all irreducible terms now written in powers of $z$ instead of $\mathrm{n}$, and the summations over differential operators builds in the far more numerous reducible diagrams. For $z$ less than the radius of convergence we expect that the activity expansion pressure, Eq. (3), will equal the virial expansion pressure, Eq. (1).

In II we considered the solution of Eqs. (3) and (4) when $S(n)$ in Eq. (1) is truncated at the second virial coefficient. When $B_{3}$ and higher virial coefficients are assumed to be zero, the corresponding cluster coefficients, $b_{j}$, are $\sim b_{2}^{j-1}$, so that the activity expansion involves an infinite number of non-zero terms. In this simple case, Eq. (3) gives directly the Mayer power series expansion in the activity, which is known to have very poor convergence properties for repulsive interactions. To overcome this problem we showed in II that summing all the terms in Eq. (3) that contain a factor 
$(\partial S / \partial z)^{2}$ yields a rational polynomial. Systematically repeating the process for terms of order $(\partial S / \partial z)^{j}$ where $j=2,3$, etc yields an infinite series of rational polynomials that are uniformly convergent. The reordered pressure equation is

$$
\begin{aligned}
& \frac{P}{k T}=z+S+\sum_{j=2}^{\infty} C_{j}, \\
& C_{2}=\frac{z x^{2} \phi}{2}, \\
& C_{3}=\frac{z x^{3} \phi^{3}}{3 !}, \\
& C_{j}=z \frac{x^{j} \phi^{2 j-3}}{j !}\left(1+\sum_{k=1}^{k=j-3} c_{j, k}(z y)^{k}\right), \\
& \phi=(1-z y)^{-1}, \\
& x=\partial S / \partial z, y=\partial^{2} S / \partial^{2} z,
\end{aligned}
$$

and the $c_{j, k}$ can be calculated according to

$$
\begin{aligned}
& c_{i, 0}=0, c_{i, 1}=0, c_{4,2}=2, \\
& c_{j, k}=k c_{j-1, k}+[2(j-1)-(k+2)] c_{j-1, k-1} .
\end{aligned}
$$

It is the appearance of the denominator in powers of $\phi$ that converts the activity series into a uniformly convergent series of rational polynomials. There are other ways 
to reorganize Eq. (3), but they are not uniformly convergent. In the present work we have extended this procedure to two-component systems. This has required that we develop a two-component generalization of Eq. (3).

\section{TWO COMPONENTS}

Our starting point for deriving the two-component generalization of Eq. (3) is again the density expansion of the canonical partition function given by

$$
\frac{P}{k T}=n_{1}+n_{2}+S\left(n_{1}, n_{2}\right)-n_{1} \frac{\partial S\left(n_{1}, n_{2}\right)}{\partial n_{1}}-n_{2} \frac{\partial S\left(n_{1}, n_{2}\right)}{\partial n_{2}}
$$

where $S\left(n_{1}, n_{2}\right)$ is the two-component generalization of (2). We showed in I (see appendix $\mathrm{A}$ in I) that when (13) is mechanically stable, $n_{1}$ and $n_{2}$ are related to the activities $z_{1}$ and $z_{2}$ according to

$$
n_{1}=z_{1} e^{\partial S / \partial n_{1}}, n_{2}=z_{2} e^{\partial S / \partial n_{2}}
$$

In order to invert Eq. $(13,14)$ to get an expansion in $z_{1}$ and $z_{2}$, similar to $(3)$, we use the method described in I. It is convenient to change variables as follows

$$
\left(n_{1}, n_{2}\right)=\left(z_{1}+u_{1}, z_{2}+u_{2}\right)
$$

where, $u_{1}=n_{1}-z_{1}$ and $u_{2}=n_{2}-z_{2}$. 
In order to illustrate the method we expand Eq. (13) to terms cubic in the variable S. This is sufficient to yield the lowest order cross-term involving the product $z_{1} z_{2}$. Higher order terms can be obtained in exactly the same way by systematically adding in the contributions of terms of order $S^{4}, S^{5} \cdots$.

From (15) we get, to order $S^{3}$,

$$
\begin{aligned}
& n_{1}=z_{1}+z_{1}\left(\frac{\partial S}{\partial n_{1}}\right)+\frac{z_{1}}{2}\left(\frac{\partial S}{\partial n_{1}}\right)^{2}+\frac{z_{1}}{6}\left(\frac{\partial S}{\partial n_{1}}\right)^{3}, \\
& n_{2}=z_{2}+z_{2}\left(\frac{\partial S}{\partial n_{2}}\right)+\frac{z_{2}}{2}\left(\frac{\partial S}{\partial n_{2}}\right)^{2}+\frac{z_{2}}{6}\left(\frac{\partial S}{\partial n_{2}}\right)^{3} .
\end{aligned}
$$

Expanding the $S\left(n_{1}, n_{2}\right)$ factors in (13) about $z_{1}$ and $z_{2}$ gives

$$
f\left(n_{1}, n_{2}\right)=f_{z}+u_{1} \frac{\partial f_{z}}{\partial z_{1}}+u_{2} \frac{\partial f_{z}}{\partial z_{2}}+\frac{u_{1}^{2}}{2} \frac{\partial^{2} f_{z}}{\partial^{2} z_{1}}+u_{1} u_{2} \frac{\partial^{2} f_{z}}{\partial z_{1} \partial z_{2}}+\frac{u_{2}^{2}}{2} \frac{\partial^{2} f_{z}}{\partial^{2} z_{2}}+\cdots
$$

where $f=S, \partial S / \partial n_{1}$ or $\partial S / \partial n_{2}$ and $f_{z}=f\left(z_{1}, z_{2}\right)$.

Now we look at the differences

$$
n_{1}-n_{1} \frac{\partial S}{\partial n_{1}} \text { and } n_{2}-n_{2} \frac{\partial S}{\partial n_{2}}
$$


From (18) we obtain

$$
\begin{aligned}
\frac{\partial S\left(n_{1}, n_{2}\right)}{\partial n_{1}}= & \frac{\partial S_{z}}{\partial z_{1}}+u_{1} \frac{\partial^{2} S_{z}}{\partial^{2} z_{1}}+u_{2} \frac{\partial^{2} S_{z}}{\partial z_{1} \partial z_{2}}+\frac{u_{1}^{2}}{2} \frac{\partial^{3} S_{z}}{\partial^{3} z_{1}}+u_{1} u_{2} \frac{\partial^{3} S_{z}}{\partial^{2} z_{1} \partial z_{2}} \\
& +\frac{u_{2}^{2}}{2} \frac{\partial^{3} S_{z}}{\partial z_{1} \partial^{2} z_{2}}, \\
\frac{\partial S\left(n_{1}, n_{2}\right)}{\partial n_{2}}= & \frac{\partial S_{z}}{\partial z_{2}}+u_{1} \frac{\partial^{2} S_{z}}{\partial z_{1} \partial z_{2}}+u_{2} \frac{\partial^{2} S_{z}}{\partial^{2} z_{2}}+\frac{u_{1}^{2}}{2} \frac{\partial^{3} S_{z}}{\partial z_{1} \partial^{2} z_{2}}+u_{1} u_{2} \frac{\partial^{3} S_{z}}{\partial z_{1} \partial^{2} z_{2}} \\
& +\frac{u_{2}^{2}}{2} \frac{\partial^{3} S_{z}}{\partial^{3} z_{2}}
\end{aligned}
$$

where $S_{z}=S\left(z_{1}, z_{2}\right)$. Substituting Eq. $(20,21)$ into (19) it is readily verified that all the terms in Eqs. (16-18) that are composed of combinations of density and activity factors cancel out. As a result, to terms cubic in $\mathrm{S}$ we get

$$
\begin{aligned}
& n_{1}-n_{1} \frac{\partial S}{\partial n_{1}}=+\frac{z_{1}}{6}\left(\frac{\partial S_{z}}{\partial z_{1}}\right)^{3}-\frac{z_{1}}{2}\left(\frac{\partial S_{z}}{\partial z_{1}}\right)-z_{1} u_{1} \frac{\partial S_{z}}{\partial z_{1}} \frac{\partial^{2} S_{z}}{\partial^{2} z_{1}} \\
& n_{2}-n_{2} \frac{\partial S}{\partial n_{2}}=\frac{z_{2}}{6}\left(\frac{\partial S_{z}}{\partial z_{2}}\right)^{3}-\frac{z_{2}}{2}\left(\frac{\partial S_{z}}{\partial z_{2}}\right)-z_{2} u_{2} \frac{\partial S_{z}}{\partial z_{2}} \frac{\partial^{2} S_{z}}{\partial^{2} z_{2}}
\end{aligned}
$$

To complete the sum in (13) we need an expression for $S\left(n_{1}, n_{2}\right)$ in terms of $z_{1}$ and $z_{2}$. Substituting for $u_{1}$ and $u_{2}$ in (18) and keeping terms of order $S^{3}$ gives 


$$
\begin{aligned}
S\left(n_{1}, n_{2}\right) & =S_{z}+z_{1}\left(\frac{\partial S_{z}}{\partial z_{1}}\right)^{2}+z_{1}\left(\frac{\partial S_{z}}{\partial z_{1}}\right)^{2} \frac{\partial^{2} S_{z}}{\partial^{2} z_{1}}+3 z_{1} z_{2} \frac{\partial S_{z}}{\partial z_{1}} \frac{\partial S_{z}}{\partial z_{2}} \frac{\partial^{2} S_{z}}{\partial z_{1} \partial z_{2}} \\
& +z_{2}\left(\frac{\partial S_{z}}{\partial z_{2}}\right)^{2}+z_{2}\left(\frac{\partial S_{z}}{\partial z_{2}}\right)^{2} \frac{\partial^{2} S_{z}}{\partial^{2} z_{2}}+\frac{z_{1}^{2}}{2}\left(\frac{\partial S_{z}}{\partial z_{1}}\right)^{2} \frac{\partial^{2} S_{z}}{\partial^{2} z_{1}}+\frac{z_{2}^{2}}{2}\left(\frac{\partial S_{z}}{\partial z_{2}}\right)^{2} \frac{\partial^{2} S_{z}}{\partial^{2} z_{2}}
\end{aligned}
$$

Now substituting Eqs. $(22,23)$ and (24) into Eq. (13) we find that the remaining negative terms cancel giving

$$
\begin{aligned}
\frac{P}{k T}= & z_{1}+z_{2}+S_{z}+\frac{z_{1}}{2}\left(\frac{\partial S_{z}}{\partial z_{1}}\right)^{2}+\frac{z_{2}}{2}\left(\frac{\partial S_{z}}{\partial z_{2}}\right)^{2}+\frac{z_{1}^{2}}{2}\left(\frac{\partial S_{z}}{\partial z_{1}}\right)^{2} \frac{\partial^{2} S_{z}}{\partial^{2} z_{1}} \\
& +\frac{z_{2}^{2}}{2}\left(\frac{\partial S_{z}}{\partial z_{2}}\right)^{2} \frac{\partial^{2} S_{z}}{\partial^{2} z_{2}}+\frac{z_{1}}{6}\left(\frac{\partial S_{z}}{\partial z_{1}}\right)^{3}+\frac{z_{2}}{6}\left(\frac{\partial S_{z}}{\partial z_{2}}\right)^{3}+z_{1} z_{2} \frac{\partial S_{z}}{\partial z_{1}} \frac{\partial S_{z}}{\partial z_{2}} \frac{\partial^{2} S_{z}}{\partial z_{1} \partial z_{2}} .
\end{aligned}
$$

Eq. (25) can be put in the more compact form

$$
\frac{P}{k T}=z_{1}+z_{2}+S_{z}+Q_{2,1}^{p}+Q_{2,2}^{p}+Q_{2,2}^{c}
$$

where,

$$
\begin{aligned}
& Q_{2,1}^{p}=\frac{1}{2 !} \sum_{i=1}^{i=2} z_{i}\left(\frac{\partial S_{z}}{\partial z_{i}}\right)^{2}, \\
& Q_{2,2}^{p}=\frac{1}{3 !} \sum_{i=1}^{i=2} z_{i} \frac{\partial}{\partial z_{i}} z_{i}\left(\frac{\partial S_{z}}{\partial z_{i}}\right)^{3},
\end{aligned}
$$




$$
Q_{2,2}^{c}=z_{1} z_{2} \frac{\partial s_{z}}{\partial z_{1}} \frac{\partial s_{z}}{\partial z_{2}} \frac{\partial^{2} S_{z}}{\partial z_{1} \partial z_{2}}
$$

the notation ' $p$ ' indicates differentiation with respect to a single variable and the notation 'c' indicates differentiation with respect to more than one variable

Following the same procedure as just described, we have worked out the contributions to the pressure from terms of order $S^{4}$ and $S^{5}$ :

$$
\begin{aligned}
& Q_{2,3}^{p}=\frac{1}{4 !} \sum_{i=1}^{i=2} z_{i} \frac{\partial}{\partial z_{i}} z_{i} \frac{\partial}{\partial z_{i}} z_{i}\left(\frac{\partial S_{z}}{\partial z_{i}}\right)^{4}, \\
& Q_{2,3}^{c}=\frac{1}{3 !} z_{1} z_{2}\left[3 \frac{\partial}{\partial z_{1}} z_{1} \frac{\partial S_{z}}{\partial z_{1}}+3 \frac{\partial}{\partial z_{2}} z_{2} \frac{\partial S_{z}}{\partial z_{2}}\right] \psi_{12}, \\
& Q_{2,4}^{p}=\frac{1}{5 !} \sum_{i=1}^{i=2} z_{i} \frac{\partial}{\partial z_{i}} z_{i} \frac{\partial}{\partial z_{i}} z_{i} \frac{\partial}{\partial z_{i}} z_{1}\left(\frac{\partial S_{z}}{\partial z_{i}}\right)^{5}, \\
& Q_{2,4}^{c}=\frac{z_{1} z_{2}}{4 !}\left[\begin{array}{c}
\frac{\partial}{\partial z_{1}} z_{1} \frac{\partial}{\partial z_{1}} z_{1}\left(\frac{\partial S_{z}}{\partial z_{1}}\right)^{2}+6 \frac{\partial}{\partial z_{1}} z_{1} \frac{\partial}{\partial z_{2}} z_{2} \frac{\partial S_{z}}{\partial z_{1}} \frac{\partial S_{z}}{\partial z_{2}} \\
+4 \frac{\partial}{\partial z_{2}} z_{2} \frac{\partial}{\partial z_{2}} z_{2}\left(\frac{\partial S_{z}}{\partial z_{2}}\right)^{2}
\end{array}\right] \psi_{12},
\end{aligned}
$$




$$
\psi_{12}=\frac{\partial S_{z}}{\partial z_{1}} \frac{\partial S_{z}}{\partial z_{1}} \frac{\partial^{2} S_{z}}{\partial z_{1} \partial z_{2}} .
$$

By induction we conclude that in general

$$
\begin{aligned}
& Q_{2, m}^{p}=\frac{1}{(m+1) !} \sum_{i=1}^{i=2} z_{i}\left(\frac{\partial}{\partial z_{i}} z_{i}\right)^{m-1}\left(\frac{\partial S_{z}}{\partial z_{i}}\right)^{m+1}, \\
& Q_{2, m}^{c}=\frac{z_{1} z_{2}}{m !} \sum_{j=1}^{j=m-1}\left(\begin{array}{c}
m \\
j
\end{array}\right)\left(\frac{\partial}{\partial z_{1}} z_{1}\right)^{m-j-1}\left(\frac{\partial}{\partial z_{2}} z_{2}\right)^{j-1}\left(\frac{\partial S_{z}}{\partial z_{1}}\right)^{m-j-1}\left(\frac{\partial S_{z}}{\partial z_{2}}\right)^{j-1} \psi_{12} .
\end{aligned}
$$

The pressure is, thus, given by

$$
\frac{P}{k T}=z_{1}+z_{2}+S_{z}+\sum_{m=1}^{m=\infty}\left(Q_{2, m}^{p}+Q_{2, m}^{c}\right)
$$

We now follow the same procedure used for the one-.component problem and sum all terms in Eq. (37) involving $\left(\partial S / \partial z_{i}\right)^{2}$. This is a much more involved process than it was for the one-component case. The result is

$$
C_{2,2}=\frac{1}{2}\left[z_{1} x_{1} \alpha_{1}+z_{2} x_{2} \alpha_{2}\right] / \phi_{2 c}
$$

where

$$
\begin{aligned}
& \alpha_{1}=x_{1}\left(1-t_{2}\right)+z_{2} x_{2} y_{12}, \\
& \alpha_{2}=x_{2}\left(1-t_{1}\right)+z_{1} x_{1} y_{12},
\end{aligned}
$$




$$
\begin{aligned}
& y_{i j}=\partial^{2} S / \partial z_{i} \partial z_{j}, \quad t_{k}=z_{k} y_{k k}, \\
& \phi_{2 c}=1-z_{1} y_{11}-z_{2} y_{22}+z_{1} z_{2}\left(y_{11} y_{12}-y_{12}^{2}\right) .
\end{aligned}
$$

Eq. (38) is again a rational polynomial where $\phi_{2 c}$ is the two-component generalization of $\phi$. Carrying out the sums to obtain higher lying coefficients gets progressively more involved. However, we have been able to sequentially sum the infinite sets of terms that include the factor $\left(\partial S / \partial z_{i}\right)^{j}$, for $j=3,4,5$, giving

$$
\begin{aligned}
C_{2,3} & =\frac{1}{6}\left[z_{1} \alpha_{1}^{3}+z_{2} \alpha_{2}^{3}\right] / \phi_{2 c}^{3} \\
C_{2,4} & =\left\{\frac{1}{24}\left[z_{1} \alpha_{1}^{4}\left(3-3 t_{2}-2 \phi_{2 c}\right)\right]+\frac{1}{24}\left[z_{2} \alpha_{2}^{4}\left(3-3 t_{1}-2 \phi_{2 c}\right)\right]\right. \\
& \left.+\frac{1}{4} y_{12} z_{1} z_{2} \alpha_{1}^{2} \alpha_{2}^{2}\right\} / \phi_{2 c}^{5} \\
C_{2,5}= & \left\{\frac{1}{5 !} z_{1} \alpha_{1}^{5}\left[\left(15\left(1-t_{2}\right)^{2}-20 \phi_{2 c}\left(1-t_{2}\right)+6 \phi_{2 c}^{2}\right)\right]\right. \\
& \quad+\frac{1}{5 !} z_{2} \alpha_{2}^{5}\left[\left(15\left(1-t_{1}\right)^{2}-20 \phi_{2 c}\left(1-t_{1}\right)+6 \phi_{2 c}^{2}\right)\right] \\
& +\frac{1}{12} y_{12} z_{1} z_{2} \alpha_{1}^{2} \alpha_{2}^{2}\left[\alpha_{2}\left(\left(1-t_{1}\right)\left(1+2 t_{2}\right)+2 y_{12}^{2} z_{1} z_{2}\right)\right. \\
& \left.+\alpha_{1}\left(\left(1-t_{2}\right)\left(1+2 t_{1}\right)+2 y_{12}^{2} z_{1} z_{2}\right)\right] \\
& \left.+\frac{1}{8} y_{12}^{2} z_{1} z_{2}\left(z_{1} \alpha_{1}^{3}+z_{2} \alpha_{2}^{3}\right)\right\} / \phi_{2 c}^{7}
\end{aligned}
$$

The higher $C_{2, j}$ coefficients can also be obtained as rational polynomials, but become too complex to be of practical value 
Knowing the $C_{2, j}$ coefficients up to $j=5$ is not sufficient to give accurate results over the entire region where the second virial expansion is the dominant term. However, we have found that the $C_{2, j}$ coefficients in Eq. (43-45) are well approximated by adding modified forms of the one-component $C_{j}$ coefficients for each component. Explicitly, the first few coefficients are.

$$
\begin{aligned}
& C_{2,2}^{a}=\frac{z_{1} x_{1}^{2}}{2 \phi_{1}}+\frac{z_{2} x_{2}^{2}}{2 \phi_{2}}, \\
& C_{2,3}^{a}=\frac{z_{1} x_{1}^{3}}{6 \phi_{1}^{3}}+\frac{z_{2} x_{2}^{3}}{6 \phi_{2}^{3}}, \\
& C_{2,4}^{a}=\frac{z_{1} x_{1}^{4}}{24 \phi_{1}^{4}}\left(1+2\left(\phi_{1}-1\right)\right)+\frac{z_{2} x_{2}^{4}}{24 \phi_{2}^{4}}\left(1+2\left(\phi_{2}-1\right)\right),
\end{aligned}
$$

$\vdots$

$$
\begin{aligned}
\phi_{i} & =1-\sum_{j=1}^{2} z_{i} y_{i j}, \\
x_{i} & =\frac{\partial S_{z}}{\partial z_{i}} .
\end{aligned}
$$

In order to couple the one-component terms in Eqs. (46-48), we have replaced $z_{1} y_{1}$ in the first component by $\sum_{j=1}^{2} z_{1} y_{1 j}$ and $z_{2} y_{2}$ in the second component by $\sum_{j=1}^{2} z_{2} y_{2 j}$.

Using a combination of the exact and approximate coefficients gives for the pressure

$$
\frac{P}{k T}=z_{1}+z_{2}+S+\sum_{j=2}^{j_{x}} C_{2, j}+\sum_{j=j_{x}+1}^{j_{\max }} C_{2, j}^{a}
$$


where $j_{x}=5$ unless $j_{\max } \leq 5$ in which case, $j_{x}=j_{\max }$.

To show the connection between the exact and approximate $C_{2, j}$ we assume that $B_{11}=B_{12}=B_{22}=B$, but that $n_{1} \neq n_{2}$.

Then,

$$
x_{1}=x_{2}=2\left(z_{1}+z_{2}\right) B
$$

And

$$
C_{2,2}^{a}=C_{2,2}=2 B^{2}\left(z_{1}+z_{2}\right)^{3} /\left(1-2 B\left(z_{1}+z_{2}\right)\right)
$$

Similarly, for the same conditions, the higher lying $C_{2, j}^{a}$ can be shown to be equal to the known exact $C_{2, j}$.

We have found numerically that when the $B_{i j}$ have a wide-range of values the approximate forms of the $C_{2, j}$ still provide a good approximation. Consequently when terms through $C_{2,5}$ are not sufficient we can improve that results by using Eqs. (46-50) to approximate terms for $j>5$.

\section{NUMERICAL EXAMPLE}


The purpose of Secs. II and III was to develop an activity expansion that uniformly converges to the virial expansion to $O\left(n^{2}\right)$ for both attractive and repulsive interactions. Fig. 1 shows $P / P_{V}$ vs. $n$. It demonstrates how well Eq. (6) achieves this result for a one-component gas. For a simple example, that illustrates the method, we have assumed $B_{2}=-0.5$. In applications for real gases we use interaction potentials obtained from Hartree-Fock ${ }^{20}$ or quantum Monte-Carlo methods ${ }^{21}$ to calculate the virial coefficients. The activity was obtained from numerical iteration to satisfy the density relation

$$
n=z \frac{\partial P / k T}{\partial z}
$$

We have not used Eq. (5) to obtain the activity because it is only valid in the region where the series has converged. Otherwise, the pressure and density are inconsistent. Fig. (1) shows that as $j_{\max }$ increases, Eq. (6) uniformly converges to the second virial pressure at increasing values of the density; i.e., each increase in $j_{\max }$ by a factor of 4 results in a fixed incremental increase $\Delta n$ to the value of $n$ where $P / P_{v}$ converges to unity. Results are also shown for the Mayer activity series truncated at different orders. When only terms $O\left(z^{2}\right)$ are included, the pressure diverges from the virial result at very small values of the density reaching a value $P / P_{v}=4 / 3$ at $n=1 / 4$. No physical solutions exist at larger values of $n$. It is not shown in Fig. (1), but we note that when the calculations are repeated sequentially adding higher order terms in $z^{j}$, the behavior is different depending on whether $j$ is even or odd. In the region where $n<1 / 4$ the results 
for the even values approach the virial result from above while the results for the odd values approach it from below. At about $O\left(z^{30}\right)$ both sets of results nearly coalesce. As the number of terms in the Mayer pressure expansion is increased further, the maximum density at which real solutions exist approaches 0.2785 and $P / P_{v} \rightarrow 1$. As shown in Fig. 2 , this limit is approached very closely when more than sixty terms are included. As expected, the Mayer expansion in powers of $z$ fares very poorly.

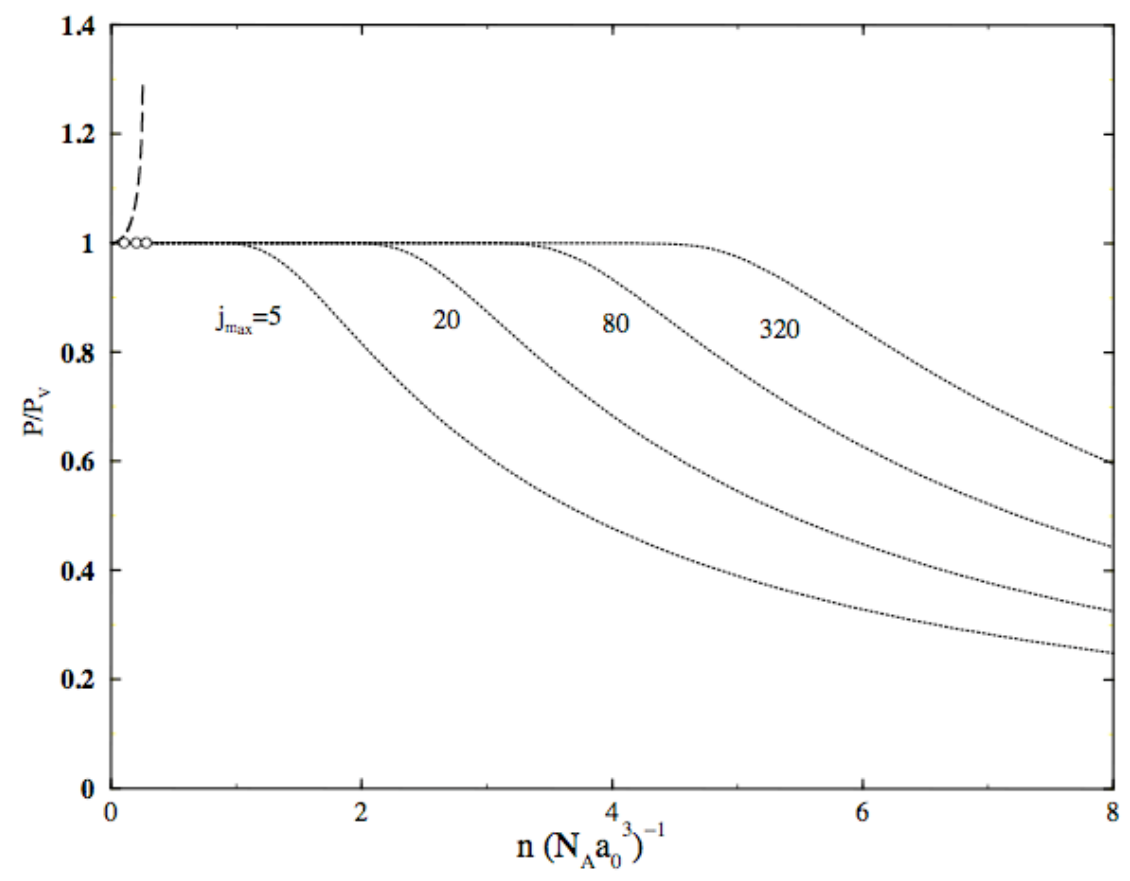

Fig. 1: Ratio of the activity expansion pressure to the second virial pressure, $P_{V}$. Pressure from Eq. (6) truncated at $j_{\max }$ (dotted). Truncated Mayer activity expansion: $O\left(z^{2}\right)$ (dashed); $O\left(z^{>60}\right)$ (open circles). 
Fig. 2 shows the ratio of the pressure from Eq. (51) to the second virial pressure of a two-component system having $B_{11}=0.125, B_{12}=0.3535, B_{22}=1.1$ and $n_{2}=2 n_{1}$. Results are shown for two different approximations of Eq. (51). Approximation 1 uses the exact $C_{2, k}$ coefficients where they are known and the approximate coefficients otherwise. Approximation 2 uses entirely the approximate coefficients. We have calculated the activities directly from a numerical solution of Eq. (51) and the density relations

$$
n_{i}=z_{i} \frac{\partial P / k T}{\partial z_{i}}, n_{i}=\left\{n_{1}, n_{2}\right\}
$$

Both versions of Eq. (51) yield results that agree closely with the virial pressure out to $n_{2}=0.5$ when $j_{x}=j \max =5$, but the deviation from unity is much smaller for approximation 1. As $j_{\max }$ is increased, both versions yield improved agreement with the virial expansion. Both approximations are uniformly convergent, but due to the use of the approximate coefficients (Eq. (46-48)) they are at variance with the virial pressure. At $n_{2}=1$, when $j_{\max }=320$, the deviation from unity for version 1 is about $4 \%$, while the deviation for approximation 2 is about $12 \%$. When $n_{2}=2 n_{1}=1, P V / N k T \approx 2.0$. In most cases, the third and higher virial coefficients make a significant contribution when the non-ideal pressure is this large. In the density range where $P V / N k T<1.6$, the second virial approximation is normally the dominant term. In this region the errors from approximation 1 are at most a few percent. Results for the Mayer activity expansion 
truncated at $O\left(z_{1}^{2}, z_{2}^{2}\right)$ are also shown in the figure. Similar to the one-component case, they diverge from the virial pressure starting at very small values of the density. The solution terminates at $n_{2}=0.1$ and $P / P_{v}=1.18$. No physical solutions exist for larger densities. Addition of higher order terms (not shown) results in behavior similar to the one-component case.

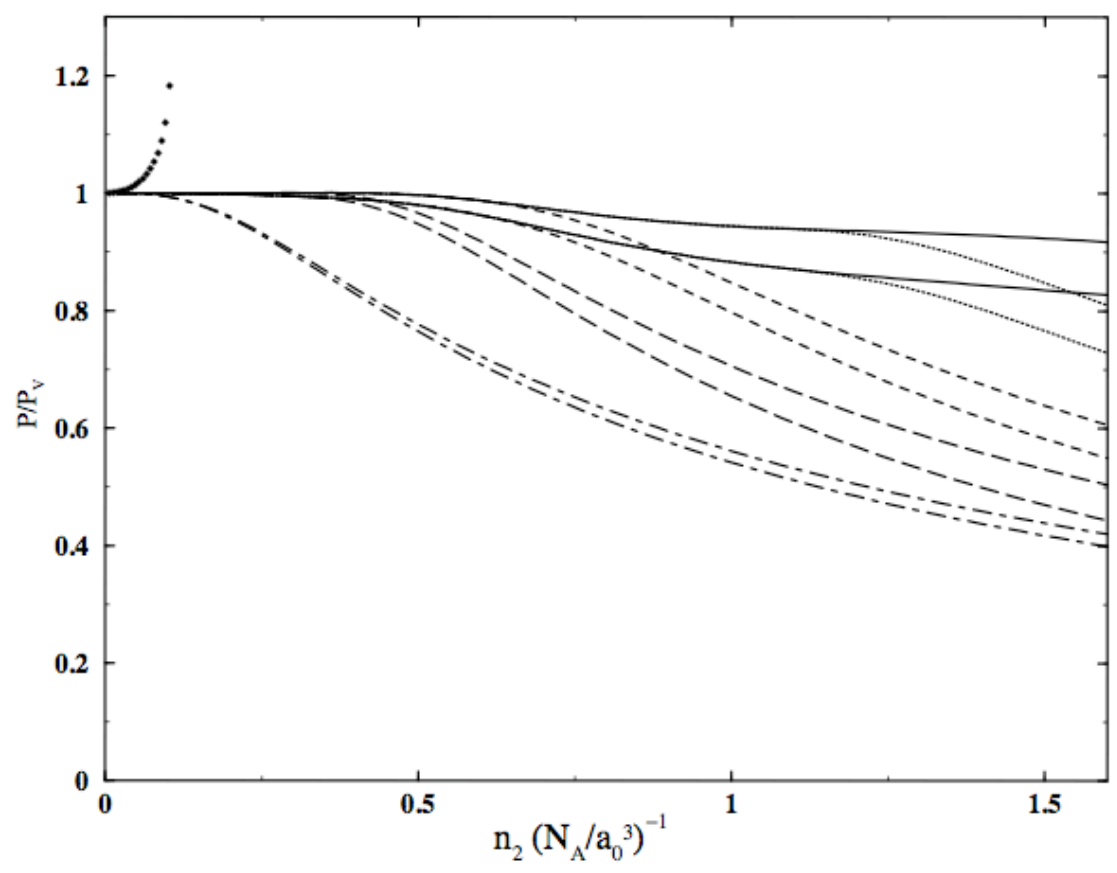


Fig. 2: $P / P_{V}$ vs. $n_{2}$ for two different approximations of Eq. (51). Approximation 1 uses the exact $C_{2, j}$ coefficients when $j \leq 5$ and the $C_{2, j}^{a}$ when $j>5$ ); approximation 2 uses entirely the

$C_{2, j}^{a}$ coefficients, i.e. $j_{x}=0$. Results are shown for $j_{\max }=2$ (dot-dashed); 5 (long-dashed); 10 (short-dashed); 40 (dotted); 320 (solid). At each value of $j_{\max }$ the approximation 1 results lies closest to unity. Results for the Mayer activity expansion truncated at $O\left(z_{1}^{2}, z_{2}^{2}\right)$ (diamonds).

\section{DISCUSSION}

We have presented a procedure for generating an activity expansion with improved convergence properties compared to the Mayer activity expansion. Herein we have only explicitly considered one and two-component systems, but the procedure can be extended to multi-component systems. We have been particularly interested in repulsive interactions since they have a very limited range of convergence for the Mayer activity expansion. Based on work started earlier we obtained expressions that generate $P / k T$ from differential operations on $S$, the sum of irreducible diagrams. We then showed that these operators could be worked out and the resulting terms regrouped into a uniformly convergent series of rational polynomials that give results equivalent to the virial expansion. Herein we have only considered the second virial approximation, which is sufficient to treat most stellar conditions. However, due to the complexity of the high lying coefficients in the resulting series, we have used an approximation that results in some small discrepancies with the second virial pressure. The procedure presented here 
can also be applied when higher virial coefficients are non-zero. We have carried this out for the case that both $B_{2}$ and $B_{3}$ are non-zero, but it is beyond the scope of the present paper to discuss it here. For applications involving more than two neutral components we have found that Approximation 2 of Eq. (51) gives accuracy similar to the twocomponent case (see Fig. 2) when $P V / N k T$ is less than about 1.6.

The activity expansion that we have described herein is of little value for applications involving purely repulsive interactions, since at best they only recover the much simpler virial expansion. However at electron volt temperatures real materials are composed of ions, atoms, and molecules. The ionic component involves the Coulomb interaction, which introduces many-body physics. Our method for treating this part of the problem was described in [1-5]. The interactions involving the atomic and molecular species are short-ranged and can be treated with the activity expansions presented herein. The complete expression used to treat partially-ionized plasmas combines the two into a single activity equation that incorporates the best features of the virial and activity expansions. The equation of state is obtained iteratively by satisfying the multicomponent density relations (Eq. (55)).

Acknowledgement: This work performed under the auspices of the U.S. Department of Energy by UC, Lawrence Livermore National Laboratory under Contract DEAC-52-07NA27344.

\section{REFERENCES:}

[1] F. J. Rogers and H.E. DeWitt, Phys. Rev. 8,1061(1973). 
[2] F. J. Rogers, Phys. Rev. A10, 2441 (1974).

[3] F. J. Rogers, Phys. Rev. A 19, 375 (1979).

[4] F. J. Rogers, Phys. Rev. A 24, 1531 (1981).

[5] F. J. Rogers, Equation of State in Astrophysics, ed. G. Chabrier \& E. Schatzmann (Cambridge University Press, Cambridge, England, 1994), p16.

[6] D. Erskine, High-Pressure Science and Technology-1993, ed. S. C. Schmidt et al., (AIP Press, New York, 1993), p125.

[7] F. J. Rogers, and D. A. Young, Phys. Rev. E 56, 5876 (1997).

[8] J. N. Bahcall, et al, Phys. Rev. Lett. 78, 171(1997).

[9] S.M. Chitre and E. N. Parker, The Dynamic Sun (Cambridge University Press, Cambridge, England, 2003), p45.

[10] J. L.Lebowitz, O. Penrose, , J. Math. Phys. 5, 841 (1964).

[11] S. Baer and J. L. Lebowitz, J. Chem Phys. 40, 3474 (1964).

[12] O. Penrose, J. Math. Phys 4, 1312 (1963).

[13] F. H. Ree, Phys. Rev. 155, 84 (1967).

[14] F. H. Ree and W. G. Hoover, J. Chem. Phys. 40, 939 (1964); 46, 4181 (1967).

[15] B. Barboy, and W. M. Gelbart, J. Chem. Phys. 71, 3063 (1979).

[16] H. L. Friedman and W. Ebeling, Theorie Elekrolytischer Flüssigkeiten, Rostocker Physikalische Manuskripte,ed. W. Ebeling and H.Künstner 4,33 (1979).

[17] M. S. Wertheim, J. Stat. Phys. 35, 19 (1984).

[18] F. J. Rogers and H. E. Dewitt, Contrib. to Plasma, Phys. 43, 355 (2003); F. J.Rogers, Contrib. to Plasma. Phys. 41, 179 (2001).

[19] E. T. Whitaker and G. N. Watson, A Course of Modern Analysis, (Cambridge 
University Press, Cambridge, England, 1952), p. 132.

[20] R. A. Aziz, F. R. W. McCourt, and C. C. K. Wong, Molec . Phys. 61, 1487 (1987).

[21] D. M. Ceperley and H. J. Partridge, J. Chem. Phys., 88, 997 (1986). 